\title{
EINE VERSCHÄRFUNG DES KOEBESCHEN VIERTELSATZES FÜR QUASIKONFORM FORTSETZBARE ABBILDUNGEN
}

\author{
REINER KÜHNAU
}

\section{Einleitung und Resultate}

Es sei $S(Q)$ die Klasse der in $z=0$ durch $w(z)=z+a_{2} z^{2}+\ldots$ normierten schlichten konformen Abbildungen $w=w(z)$ von $|z|<1$, die sich zu einer stetigen schlichten $Q$-quasikonformen Abbildung der Vollkugel mit $w(\infty)=\infty$ fortsetzen lassen. Entsprechend bestehe die Klasse $\Sigma(Q)$ aus allen in $z=\infty$ durch $w(z)=z+A_{1} z^{-1}+\ldots$ hydrodynamisch normierten schlichten konformen Abbildungen von $|z|>1$, die sich zu einer stetigen schlichten $Q$-quasikonformen Abbildung der Vollebene fortsetzen lassen. Für diese Klassen wurden jüngst in [1], [3], [5-18] mit verschiedenen Methoden spezielle Extremalprobleme betrachtet.

In [15] wurde dabei eine allgemeine Strukturformel für die durch quadratische Differentiale charakterisierten Extremalfunktionen aufgestellt, die bei Extremalproblemen vom Grötzsch-Teichmüllerschen Typ in diesen Klassen auftreten. Die Trächtigkeit dieser Strukturformel soll jetzt hier an einem Beispiel erläutert werden. Unter Verwendung der gebräuchlichen Bezeichnungen $C$ für die Eulersche Konstante $(C=0.577 \ldots)$ und $I$ und $\psi$ für die Eulersche Gamma- bzw. Psifunktion $\left(\psi=\Gamma^{\prime} / \Gamma\right)$ sowie der Abkürzung $q=(Q-1) /(Q+1)$ werden wir folgende Verschärfung des Koebeschen Viertelsatzes beweisen.

Satz 1. Für jede Abbildung aus $S(Q)$ liegt das Bild von $|z|=1$ in dem Kreisring

$$
m(Q) \leq|w| \leq M(Q)
$$

wo

(2) $m(Q)=\exp \left\{-2 C-6 \log 2-2 \psi\left(2^{-1}-(2 \pi)^{-1} \arccos q\right)-\pi Q^{-\frac{1}{2}}\right\}$,

(3) $M(Q)=\exp \left\{-2 C-6 \log 2-2 \psi\left((2 \pi)^{-1} \arccos q\right)-\pi Q^{\frac{1}{2}}\right\}$.

Ungleichung (1) ist scharf. 
Extremalfunktionen nebst anderen Darstellungen für $m(Q)$ und $M(Q)$ werden sich aus dem Beweis ergeben.

Für kleine Werte von $q$ bestehen die asymptotischen Darstellungen

$$
\begin{aligned}
& m(Q)=1-(8 / \pi) G q+q^{2} \mathfrak{P}(q), \\
& M(Q)=1+(8 / \pi) G q+q^{2} \mathfrak{P}(q),
\end{aligned}
$$

wo $G=0.915 \ldots$ die Catalansche Konstante ist und $\mathfrak{P}$ eine Potenzreihe bezeichnet. Für kleine Werte von $1-q$ (d. h. große Werte von $Q$ ) gilt

$$
\begin{aligned}
& m(Q)=\frac{1}{4}\left(1+(1-q) \mathfrak{D}\left((1-q)^{\frac{1}{2}}\right)\right), \\
& M(Q)=\exp \left\{\pi 2^{\frac{1}{2}}(1-q)^{-\frac{1}{2}}+\mathfrak{D}\left((1-q)^{\frac{1}{2}}\right)\right\} .
\end{aligned}
$$

Die Darstellungen (4) und (5) wurden schon von S. L. Kruškal' [5], [6] angegeben; vgl. auch die Verallgemeinerung von Lehto [18]. In (6) steckt für $Q \rightarrow \infty$ der klassische Koebesche Viertelsatz.

Mit Satz 1 hängt eng zusammen der folgende

Satz 2. Für die Abbildungen $w=w(z)$ der Klasse $\Sigma(Q)$ gilt die scharfe Abschätzung

$$
2 M(Q)^{-\frac{1}{2}} \leq|w(1)-w(-1)| \leq 2 m(Q)^{-\frac{1}{2}} .
$$

Durch lineare Transformation fließt hieraus weiter der

Satz 3. Für die schlichten konformen Abbildungen $W=W(Z)$ von $\operatorname{Im} Z>0$, die sich zu einer stetigen schlichten Q-quasikonformen Abbildung der Vollebene (mit $\infty \rightarrow \infty)$ fortsetzen lassen, gilt bei $\operatorname{Im} Z>0$ die scharfe Abschätzung

$$
M(Q)^{-\frac{1}{2}} \leq\left|(\operatorname{Im} Z) \frac{W^{\prime}(Z)}{W(Z)-W(\operatorname{Re} Z)}\right| \leq m(Q)^{-\frac{1}{2}}
$$

\section{Die Strukturiormel}

Der Beweis der genannten Sätze stützt sich wesentlich auf die folgende Strukturformel, die in [15] angegeben wurde. Sei dazu zunächst $w=w(z)$ allgemein eine stetige schlichte Abbildung der Vollkugel auf sich mit $w(\infty)=\infty$, die für $|z|>1$ konform ist und die in $|z|<1$ gelegenen infinitesimalen Kreise in infinitesimale Ellipsen des Achsenverhältnisses $Q>1$ transformiert, wobei in Richtung der großen Achsen $\mathfrak{Q}(w) d w^{2} \geq 0$ sei mit einer vorgegebenen rationalen Funktion $\mathfrak{Q}(w)$. Dann gilt 
$(10)$

$$
\int(\mathfrak{Q}(w))^{\frac{1}{2}} d w= \begin{cases}F(z) & \text { für }|z| \geq 1, \\ \left(1-q^{2}\right)^{-1}[\bar{G}(1 / \bar{z})+q G(1 / \bar{z})] & \text { für }|z| \leq 1\end{cases}
$$

mit

$$
\begin{aligned}
& F(z)=-\left(1-q^{2}\right)^{-\frac{1}{2}} \int h^{\frac{1}{2}} \sin I d z, \\
& G(z)=\int h^{\frac{1}{2}} \cos I d z,
\end{aligned}
$$

und

$$
I(z)=\left(1-q^{2}\right)^{\frac{1}{2}} \int^{z}(k / h) d z .
$$

Hier sind $h$ und $k^{2}$ (in [15] näher charakterisierte) rationale Funktionen, für die $z^{2} h$ bzw. $z^{3} k$ auf $|z|=1$ reell ist. Dazu soll noch eine ergänzende Relation angegeben werden, die sich aus der Übereinstimmung der beiden in (10) stehenden Ausdrücke für $|z|=1$ ergibt. Zu diesem Zweck setzen wir $z=e^{i t}$ und differenzieren die so entstehende Gleichung nach $t$, was zu

$$
\left(1-q^{2}\right)^{\frac{1}{2}} \sin I= \pm \overline{\cos I}-q \cos I, \quad|z|=1,
$$

führt. Hierbei steht das obere Zeichen bei $z^{2} h>0$, das untere Zeichen bei $z^{2} h<0$. Trennung dieser Gleichung in Real- und Imaginärteil führt (über die Anwendung von Additionstheoremen) auf

$$
\begin{aligned}
& \left(1-q^{2}\right)^{\frac{1}{2}} \sin (\operatorname{Re} I)= \pm \cos (\operatorname{Re} I)-q \cos (\operatorname{Re} I), \\
& \left(1-q^{2}\right)^{\frac{1}{2}} \cos (\operatorname{Re} I)= \pm \sin (\operatorname{Re} I)+q \sin (\operatorname{Re} I) .
\end{aligned}
$$

Letztere beide Gleichungen sind ersichtlich äquivalent und liefern die gesuchte Gleichung

$$
q= \pm \cos (2 \operatorname{Re} I(z)), \quad|z|=1,
$$

mit der genannten Vorzeichenregelung.

\section{Beweise der Sätze $1-3$}

Satz 1 folgt in bekannter Weise nach Stürzung und einer Quadratwurzeloperation aus Satz 2. Satz 3 folgt aus Satz 2 durch lineare Transformation von $|z|>1$ in die obere Halbebene, wobei $z=\infty$ in $Z, z=1$ in $\infty$ und $z=-1$ in $\operatorname{Re} Z$ übergeht, und lineare Transformation der $w$-Ebene, wobei $w(1)$ in $\infty$ übergeht. Deshalb können wir uns im folgenden auf den Beweis von Satz 2 konzentrieren.

Betrachten wir dazu also in der Klasse $\Sigma(Q)$ die Extremalprobleme, das Minimum bzw. Maximum von $|w(1)-w(-1)| \mathrm{zu}$ bestimmen. Nach den allgemeinen Bemerkungen am Anfange von [7] ist die Extremalfunktion hierzu jeweils eindeutig bestimmt und von der Art, wie oben am Anfange von $\S 2$ beschrieben. Im hier vorliegenden Falle ist 


$$
\mathfrak{Q}(w) d w^{2}= \pm d w^{2} /\left(w^{2}-w_{1}^{2}\right), \quad w_{1}=w(1)=-w(-1)>0,
$$

da sich dieses quadratische Differential nach H. Grötzsch (vgl. die Darstellung in [4, Chapter VI]) auch bei konformen Abbildungen einstellt. Hierbei steht - wie auch im folgenden stets - das obere Zeichen im Falle des Minimumproblems, das untere Zeichen im Falle des Maximumproblems. Wegen

$$
\int\left(w^{2}-w_{1}^{2}\right)^{-\frac{1}{2}} d w=\operatorname{ar} \cosh \left(w / w_{1}\right)+\text { const }=\log w+\mathfrak{D}(1 / w)
$$

ergibt sich aus (10) und der Normierung unserer Abbildung in $z=\infty$ dort der Entwicklungstypus $\quad F^{\prime}(z)=( \pm 1)^{\frac{1}{2}} \log z+\mathfrak{P}(1 / z), \quad F^{\prime}(z)=( \pm 1)^{\frac{1}{2}} /$ $z+\ldots, \quad F^{\prime \prime}(z)=-( \pm 1)^{\frac{1}{2}} / z^{2}+\ldots, \quad F^{\prime 2}(z)= \pm 1 / z^{2}+\ldots, \quad G(z)=$ $\mathfrak{P}(1 / z), G^{\prime}(z)=\mathfrak{P}(1 / z) / z^{2}, G^{\prime \prime}(z)=\mathfrak{P}(1 / z) / z^{3}$, also nach [15] (Formel $\left.(6)\right)$

$$
z^{2} h= \pm\left(1-q^{2}\right)+\ldots, \quad z^{6} k^{2}=\mathfrak{P}(1 / z) / z^{2} \quad \text { mit } \mathfrak{P}(0) \neq 0 .
$$

Nach Formel (12) in [15] folgt daraus die Darstellung

$$
z^{2} h=i B_{1}(z+1) /(z-1)+i B_{2}(z-1) /(z+1)+D,
$$

$B_{1}, B_{2}, D$ reelle Konstanten. Vergleich mit (15) zeigt, dass $\pm\left(1-q^{2}\right)=$ $i\left(B_{1}+B_{2}\right)+D$ ist, also $B_{1}+B_{2}=0, \quad D= \pm\left(1-q^{2}\right)$. Damit landen wir bei

$$
z^{2} h= \pm\left(1-q^{2}\right)+i B_{1} 4 z /\left(z^{2}-1\right),
$$

was weiter $B_{1}=0$ nach sich zieht, da $h$ nach (6) in [15] gerade sein muß. Wir haben somit

$$
z^{2} h= \pm\left(1-q^{2}\right)
$$

Nach Formel (14) in [15] folgt nun weiter in Berücksichtigung der Entwicklung (15)

$$
z^{6} k^{2}=A z^{4}(z-1)^{-4}(z+1)^{-4} \prod_{1}^{2}\left(z-k_{v}\right)\left(1-\bar{k}_{v} z\right) / z, \quad A>0, \quad\left|k_{v}\right| \geq 1 .
$$

Hier müssen beide Stellen $k_{v}$ auf dem Einheitskreis liegen, da andernfalls ein Widerspruch dazu folgt, daß $k(z)$ eine gerade und (nach [15]) auch eindeutige Funktion ist. Das bedeutet weiter $k_{2}=-k_{1}$ und $\left|k_{1}\right|=1$, also

$$
\begin{aligned}
z^{6} k^{2} & =-A \bar{k}_{1}^{2} z^{2}\left(z^{2}-k_{1}^{2}\right)^{2} /\left(z^{2}-1\right)^{4}, \\
z^{2} k & =(-A)^{\frac{1}{2}} \vec{k}_{1}\left(z^{2}-k_{1}^{2}\right) /\left(z^{2}-1\right)^{2} .
\end{aligned}
$$

Für positiv reelle $z$ sind $F^{\prime}$ und $F^{\prime \prime}$ imaginär, $G^{\prime}$ und $G^{\prime \prime}$ reell, also ist $k(z)$ imaginär, so daß auch der Parameter $k_{1}$ reell sein muß und z.B. $=$ +1 gesetzt werden kann. Dies liefert dann endgültig 


$$
z^{2} k(z)=(-A)^{\frac{1}{2}} /\left(z^{2}-1\right) .
$$

Setzt man dies und (16) in (11) ein, so kommt

(18) $F(z)=-( \pm 1)^{\frac{1}{2}} \int z^{-1} \sin (i c \log ((z-1) /(z+1))+$ const $) d z, \quad c>0$.

Der Vergleich mit der oben nach (14) angegebenen Entwicklung von $F$ in Umgebung von $z=\infty$ liefert für die in (18) auftretende Konstante $\sin ($ const $)= \pm 1, \quad$ const $=\pi / 2+n \pi \quad(n$ ganzzahlig $) . \quad$ Demnach ist bei geeigneter Zweigwahl der Quadratwurzel

$$
\begin{aligned}
F(z) & =-( \pm 1)^{\frac{1}{2}} \int z^{-1} \cos (i c \log ((z-1) /(z+1))) d z= \\
& =-( \pm 1 / 4)^{\frac{1}{2}} \int z^{-1}\left[(z-1)^{c}(z+1)^{-c}+(z+1)^{c}(z-1)^{-c}\right] d z, \\
G(z) & =\left( \pm\left(1-q^{2}\right)\right)^{\frac{1}{2}} \int z^{-1} \sin (i c \log ((z-1) /(z+1))) d z= \\
& =i\left( \pm\left(1-q^{2}\right) / 4\right)^{\frac{1}{2}} \int z^{-1}\left[(z-1)^{c}(z+1)^{-c}-(z+1)^{c}(z-1)^{-c}\right] d z .
\end{aligned}
$$

Für $|z| \geq 1$ ist also nach (10) und (14) die gesuchte Extremalfunktion

$$
w(z)=w_{1} \cosh \left(\frac{1}{2} \int_{1}^{z} z^{-1}\left[(z-1)^{c}(z+1)^{-c}+(z+1)^{c}(z-1)^{-c}\right] d z\right) .
$$

Hier lautet die Entwicklung in $z=\infty$

$$
w(z)=w_{1} \cosh (\log z+E+\ldots)=\frac{1}{2} w_{1} e^{E} z+\mathfrak{P}(1 / z) .
$$

Das gestattet den Koeffizientenvergleich

$$
w_{1}=2 e^{-E}
$$

mit

$$
E=\frac{1}{2} \int_{1}^{\infty} z^{-1}\left[(z-1)^{c}(z+1)^{-c}+(z+1)^{c}(z-1)^{-c}-2\right] d z
$$

(zu integrieren etwa längs der positiven reellen Achse). Damit hätten wir eine mögliche Darstellung der Schranken in (8), d. h. für $m(Q)$ und $M(Q)$ gefunden, nachdem noch der (unten unter (22), (23) angegebene) Wert für $c$ eingesetzt worden ist. Man erhält noch die Gestalt (2) bzw. (3), indem man in (20) die Substitution $t=(z-1) /(z+1)$ ausführt (vgl. z.B. [2]):

$$
\begin{aligned}
E & =\int_{0}^{1}\left(t^{c}+t^{-c}-2\right)\left(1-t^{2}\right)^{-1} d t= \\
& =\frac{1}{2}[\psi(1 / 2)-\psi((1+c) / 2)+\psi(1 / 2)-\psi((1-c) / 2)]= \\
& =\psi(1 / 2)-\frac{1}{2}[\psi((1+c) / 2)+\psi((1-c) / 2)]= \\
& =-C-\log 4-\psi((1-c) / 2)-(\pi / 2) \operatorname{tg}(\pi c / 2)
\end{aligned}
$$


Der Wert für $c$ ergibt sich aus (12):

$$
\begin{array}{ll}
c=1-(1 / \pi) \operatorname{arc} \cos q & \text { im Falle des Minimumproblems, } \\
c=(1 / \pi) \operatorname{arc} \cos q & \text { im Falle des Maximumproblems }
\end{array}
$$

(um zu dieser Zweigfestlegung bei are cos zu kommen, verwendet man die Konvergenz in (21) oder einen der Grenzfälle $q=0$ bzw. $q=1$ ). Nachdem so (2) und (3) gefunden sind, erhält man leicht auch (nach einigen Rechnungen z.B. nach [2]) die angegebenen asymptotischen Entwicklungen.

\section{Zusatzbemerkungen}

Es läßt sich nun auch leicht der genaue Wertebereich der Größe $\log (w(1)-w(-1))$ in der Klasse $\Sigma(Q)$ bzw. der Größe log $w(1)$ in der Klasse $S(Q)$ angeben. Nach den einleitenden Bemerkungen in [7] ist nämlich wie im konformen Falle bei H. Grötzsch (vgl. z.B. die Darstellung in [4, Chapter VI]) dieser genaue Wertebereich eine abgeschlossene Kreisscheibe, für die in Satz 2 bzw. 1 Diametralpunkte bestimmt worden sind.

Weiter kann man im Anschluß an obige Untersuchungen in der Klasse $\Sigma(Q)$ auch das zu Satz 2 analoge Extremalproblem min $\left|w\left(z_{1}\right)-w\left(z_{2}\right)\right|$ bzw. $\max \left|w\left(z_{1}\right)-w\left(z_{2}\right)\right|$ betrachten, wenn $z_{1}$ und $z_{2}$ irgendwelche Punkte auf $|z|=1$ sind. (Für beliebige Punkte $z_{1}$ und $z_{2}$ in der $z$-Ebene ist auch eine Behandlung mit der Strukturformel von [15] möglich, was aber einen ganz erheblich größeren Formelaufwand erfordert.) So läßt sich auch diskutieren, welches der größte Wert für den anfallenden Maximalwert ist. Das liefert also in der Klasse $\Sigma(Q)$ das Maximum des Durchmessers des Bildes von $|z|=1$. (Dieses Maximum ist vermutlich gleich der rechten Seite von (8).) Durch eine Stürzung entsteht dann auch die Lösung der Aufgabe, das Funktional $\min _{|z|=1}|w(z)|$ am kleinsten zu machen in der Klasse der Abbildungen von $S$, die eine $Q$-quasikonforme Fortsetzung gestatten. (Der Unterschied zum Extremalproblem entsprechend dem linken Teile der Ungleichung (1) besteht im Wegfallen der Bedingung $w(\infty)=\infty$.)

\section{Literatur}

[1] Alenicyn, JU. E.: Некоторые теоремы площадей для аналитических функций с квазиконформным продолжением - Dokl. Akad. Nauk SSSR 215, 1974, 1025-1028 und Mat. Sb. 94, 1974, 114-125.

[2] Gröbner, W., und N. Hofreiter: Integraltafel, 2. Teil, Bestimmte Integrale. Springer-Verlag, Wien und Innsbruck, 1950.

[3] Guthjanskir, V. JA.: O принципе площадей для одного класса квазиконформных отображений. - Dokl. Akad. Nauk SSSR 212, 1973, 540-543. Übersetzung: On the area principle for a class of quasi-conformal mappings. - Soviet Math. Dokl. 14, 1973, 1401-1406. 
[4] Jenkins, J. A.: Univalent functions and conformal mapping. - Springer-Verlag, Berlin - Göttingen - Heidelberg, 1958.

[5] KRUSKAL', S. L.: Некоторые экстремальные задачй для однолистных аналитических функцнй. - Dokl. Akad. Nauk SSSR 182, 1968, 754-757. Übersetzung: Some extremal problems for schlicht analytic functions. Soviet Math. Dokl. 9, 1968, 1191-1194.

[6] -»- Некоторые экстремальные задачи для конформных и квазиконформных отображений. - Sibirsk. Mat. Ž. 12, 1971, 760-784. Übersetzung: Some extremal problems for conformal and quasiconformal mappings. - Siberian Math. J. 12, 1971, 541-559.

[7] KÜHNAU, R.: Wertannahmeprobleme bei quasikonformen Abbildungen mit ortsabhängiger Dilatationsbeschränkung. - Math. Nachr. 40, 1969, 1-11.

[8] - - Einige Extremalprobleme bei differentialgeometrischen und quasikonformen Abbildungen. II. - Math. Z. 107, 1968, 307-318.

[9] -»- Verzerrungssätze und Koeffizientenbedingungen vom Grunskyschen Typ für quasikonforme Abbildungen. - Math. Nachr. 48, 1971, 77-105.

[10] - - Koeffizientenbedingungen bei quasikonformen Abbildungen. - Ann. Univ. Mariae Curie-Skłodowska Sect. A 22-24, 1968-1970, 105-111.

[11] - - Eine funktionentheoretische Randwertaufgabe in der Theorie der quasikonformen Abbildungen. - Indiana Univ. Math. J. 21, 1971, 1-10.

[12] - - Weitere elementare Bemerkungen zur Theorie der konformen und quasikonformen Abbildungen. - Math. Nachr. 51, 1971, 377-382.

[13] -»- Zum Koeffizientenproblem bei den quasikonform fortsetzbaren schlichten konformen Abbildungen. - Math. Nachr. 55, 1973, 225-231.

[14] - - Zur Abschätzung der Schwarzschen Ableitung bei schlichten Funktionen. Math. Nachr. 59, 1974, 195-198.

[15] - - Zur analytischen Darstellung gewisser Extremalfunktionen der quasikonformen Abbildung. - Math. Nachr. 60, 1974, 53-62.

[16] Kühnau, R., und B. Dittmar: Einige Folgerungen aus den Koeffizientenbedingungen vom Grunskyschen Typ für schlichte quasikonform fortsetzbare Abbildungen. - Math. Nachr. (im Druck).

[17] Lehto, O.: Schlicht functions with a quasiconformal extension. - Ann. Acad. Sci. Fenn. Ser. A I 500, 1971, 1-10.

[18] - - Quasiconformal mappings and singular integrals. - Erscheint in Symposia Math. Istituto Nazionale di Alta Matematica Roma.

Martin-Luther-Universität Halle-Wittenberg

Mathematisches Institut

Halle an der Saale

DDR

Eingegangen am 9. September 1974 